\title{
The Generation of Random Variates From a Relativistic Maxwellian Distribution
}

\author{
M. Swisdak ${ }^{1, a)}$ \\ Institute for Research in Electronics and Applied Physics, University of Maryland, College Park, \\ MD 20742
}

(Dated: 16 October 2018)

A procedure for generating random variates from a relativistic Maxwellian distribution with arbitrary temperature and drift velocity is presented. The algorithm is based on the rejection method and can be used to initialize particle velocities in kinetic simulations of plasmas and gases.

PACS numbers: 52.65.-y,52.65.Rr,52.27.Ny

\section{INTRODUCTION}

Kinetic simulations of plasmas and gases follow the trajectories of individual particles as they evolve from an initial state, often one for which the velocities are random variates drawn from a specified distribution function. For systems with negligible relativistic and quantum effects, that distribution is often the Maxwell-Boltzmann (Maxwellian). Generating initial particle velocities is then straightforward since a non-relativistic Maxwellian separates, with each coordinate governed by a Gaussian (normal) distribution for which quick and simple methods of generating variates are known ${ }^{1}$. Drifting distributions (those with non-zero average velocities) are only slightly more complicated since Galilean transformations apply in the non-relativistic limit.

Special relativity, however, does not permit velocities greater than the speed of light $c$ and is thus incompatible with a Maxwellian distribution that predicts a non-zero probability for every velocity. Jüttner ${ }^{2}$ introduced the first relativistic generalization of the Maxwellian, basing the derivation on Boltzmann's expression for the probability of finding a system in a state with energy $E$,

$$
f=\frac{1}{Z(T)} e^{-E / k_{B} T}
$$

Here $f, k_{B}, T$, and $Z(T)$ are the distribution function, Boltzmann's constant, the temperature, and the partition function, respectively. In special relativity the total energy for a particle with rest mass $m$ is $E=\gamma m c^{2}$, although it is often more useful to consider the kinetic energy $(\gamma-1) m c^{2}$, which only differs by a factor that can be absorbed into the normalization. Defining

$$
A=\frac{m c^{2}}{k_{B} T}
$$

and henceforth writing velocities, momenta, and energies in dimensionless units - $u=u / c, p=p / m c$, and $E=$

\footnotetext{
a) Electronic mail: swisdak@umd.edu
}

$E / m c^{2}$ - gives the distribution

$$
f(\mathbf{p})=\frac{1}{Z(T)} e^{-A(E(\mathbf{p})-1)}
$$

Perhaps surprisingly, the correct form of $Z$ is still being debated ${ }^{3-5}$, but, fortunately for the purposes of variate generation, it only contributes a multiplicative factor that, crucially, does not depend on the momentum. Hence, for any variate-generating algorithm in which only ratios of $f$ appear, $Z$ (and, in fact, any multiplicative pre-factors that do not depend on the momentum) can be dropped.

Neither Jüttner's distribution nor equation 3, from which it can be derived, are Lorentz-covariant (i.e., expressed solely in terms of scalars, 4 -vectors, etc.). Synge $e^{6}$ was perhaps the first to suggest a covariant form, the only features of which that matter for variate generation are: (1) $E$ changes between reference frames via a Lorentz transformation, and (2) The form of the variation in $T$ between reference frames is known. This work assumes $T$ is an invariant, but the formalism directly carries over to the case $T=T(u)$, where $u$ is the reference frame velocity.

Both the isotropic and drifting relativistic Maxwellians are special cases of a class known as generalized hyperbolic distributions that was first used by BarndorffNielsen to describe measurements of aeolian sand grains ${ }^{7}$. Although the original work considered univariate (i.e., one-dimensional) distributions, multivariate versions quickly followed ${ }^{8,9}$, as did algorithms for generating random hyperbolic variates via a mixture of normallydistributed variates and variates of the generalized inverse Gaussian distribution ${ }^{10}$. The semi-heavy tails of generalized hyperbolic distributions make them useful for modelers of financial markets and has spurred the development of several numerical packages for generating variates. However they tend to work efficiently only for a narrow range of temperatures and drift speeds.

Pozdnyakov et al,$\underline{11,12}$ describe an algorithm specific to the relativistic Maxwellian distribution, albeit restricted to the isotropic case, that separately considers the large and small $A$ limits. Its efficiency (defined as how often the procedure generates an acceptable variate), varies from nearly $100 \%$ for $A<1$ to $33 \%$ for $A \rightarrow \infty$. The al- 
gorithm presented here is slightly more complicated but also more general, as it works efficiently $(\approx 90 \%$ for all $A$ in the isotropic case) for nearly arbitrary drift speeds and temperatures

Sections III and III address the generation of variates for stationary and drifting Maxwellians, respectively. Both use an algorithm originally due to Devroye $\underline{13}$ that has been adapted for this work and is described in the Appendix.

\section{STATIONARY MAXWELLIAN}

Using the rest-frame relationship between energy and momentum, equation 3 becomes

$$
f(\mathbf{p})=e^{-A\left(\sqrt{1+|\mathbf{p}|^{2}}-1\right)}
$$

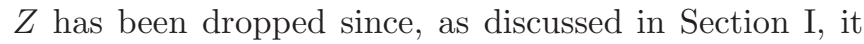
does not affect the generation of variates. This distribution function is isotropic and can be re-written more conveniently in terms of $p=|\mathbf{p}|$ by moving to spherical coordinates, integrating over the angle variables, and dropping the constant pre-factors:

$$
f(p)=p^{2} e^{-A\left(\sqrt{1+p^{2}}-1\right)}
$$

By keeping the seemingly extraneous $e^{A}$ term, $f$ can be written in an equivalent form

$$
f(p)=p^{2} e^{-A p^{2} /\left(1+\sqrt{1+p^{2}}\right)}
$$

that avoids numerical problems in the exponent for small $p$. Once a $p$ has been generated from this distribution, it can be uniformly distributed over the surface of a sphere (see, e.g., Knop $\stackrel{14}{ }$ ) to give individual Cartesian components.

Since $f$ is $\log$-concave, i.e., $(\log f)^{\prime \prime} \leq 0$ for all $p$, the algorithm described in the Appendix can be used to generate random variates. The only additional information needed is the mode

$$
p_{m}^{2}=\frac{2}{A^{2}}\left(1+\sqrt{1+A^{2}}\right)
$$

Figure 1 demonstrates the results for $A=10^{12}$ (roughly typical of water vapor at room temperature), $10^{6}$ (keV protons), 1 ( $\mathrm{MeV}$ electrons), and $10^{-6}$ ( $\mathrm{TeV}$ electrons). The top portion of each panel compares the expected $f$ from equation 6 to a histogram of the generated variates. The two curves, each normalized to unit area, are nearly indistinguishable and so the bottom portion of each panel plots the ratio of the histogram to the expected value on a logarithmic scale ranging from $1 / 2$ to 2. Because the variate-generating algorithm is based on the rejection method, not every generation attempt succeeds. For each panel $10^{6}$ attempts were made, with the success rate varying only slightly with $A$, from $88 \%$ for $A=10^{12}$ to $90 \%$ for $A=10^{-6}$. Although the algorithm
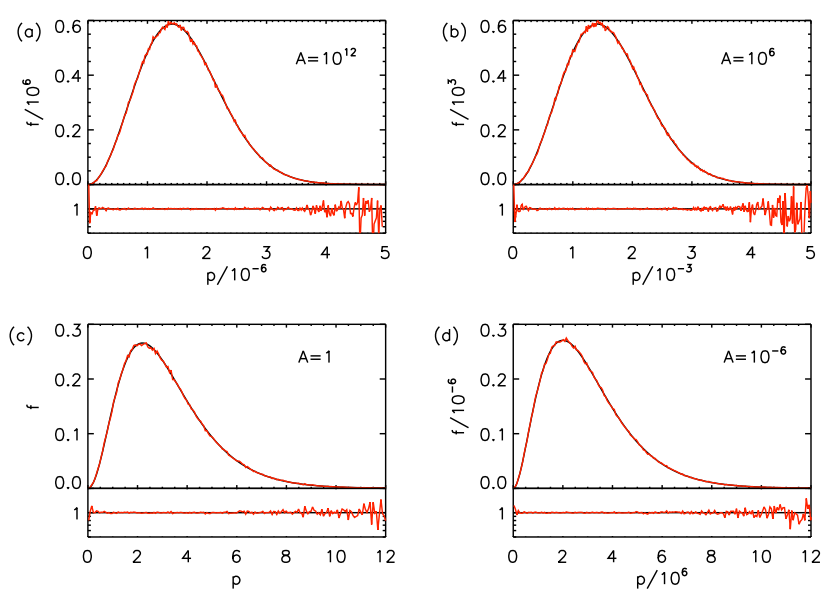

FIG. 1. Comparison between the theoretical distribution of $p$ from equation 6 and the generated random variates for isotropic relativistic Maxwellians with different values of $A$. The top plot in each of the four panels shows the theoretical distribution in black with the computed distribution overplotted in red. The bottom plot shows the ratio of the computed to theoretical distributions on a log scale ranging from $1 / 2$ to 2 . Note the different axis scales for each panel.

produces excellent results for all $A$, note that in systems with $A \lesssim 1$ pair production can occur and any distribution that assumes a constant particle number, such as the $f$ of equation 5 cannot be completely accurate.

In the non-relativistic limit the exponential term in equation 5 reduces to $\exp \left(-A p^{2} / 2\right)$, which implies that varying $A$ and $1 / p^{2}$ by the same factor will produce similar distributions. This effect can be observed in panels (a) and (b) which, aside from the axis scales, are nearly identical. Panels (c) and (d) cover the relativistic limit in which the exponential term reduces to $\exp (-A p)$ so that jointly scaling $A$ and $1 / p$ produces the same effect.

\section{DRIFTING MAXWELLIAN}

In a frame moving with velocity $\mathbf{u}$ with respect to the rest frame the Lorentz-transformed energy of a particle is

$$
E^{\prime}=\gamma_{u}(E-\mathbf{u} \cdot \mathbf{p})
$$

where $\gamma_{u}=1 / \sqrt{1-u^{2}}$. Equation 3 then becomes

$$
f(\mathbf{p})=e^{-A\left(\gamma_{u} \sqrt{1+|\mathbf{p}|^{2}}-\gamma_{u} \mathbf{u} \cdot \mathbf{p}-1\right)}
$$

To proceed, define a coordinate system with axes parallel and perpendicular to the drift velocity, $\left(p_{\|}, \mathbf{p}_{\perp}\right)$. The anisotropic, non-separable form of $f$ means that the perpendicular momenta must be generated conditional on the value of $p_{\|}$. Moving to cylindrical coordinates and integrating over the perpendicular coordinates gives the 
distribution for $p_{\|}$

$$
f\left(p_{\|}\right)=\int_{0}^{2 \pi} \int_{0}^{\infty} p_{\perp} e^{-A\left(\gamma_{u} \sqrt{1+p_{\|}^{2}+\left|\mathbf{p}_{\perp}\right|^{2}}-\gamma_{u} u p_{\|}-1\right)} \mathrm{d} p_{\perp} \mathrm{d} \theta
$$

Ignoring, as usual, multiplicative scaling coefficients leaves

$$
f\left(p_{\|}\right)=\left(1+A \gamma_{u} \sqrt{1+p_{\|}^{2}}\right) e^{-A\left(\gamma_{u} \sqrt{1+p_{\|}^{2}}-\gamma_{u} u p_{\|}-1\right)}
$$

and it is again useful to express the exponent in a numerically stable form,

$$
f\left(p_{\|}\right)=\left(1+A \gamma_{u} \gamma_{\|}\right) e^{-A\left(p_{\|}-p_{u}\right)^{2} /\left(\gamma_{\|} \gamma_{u}+p_{\|} p_{u}+1\right)}
$$

where $p_{u}=\gamma_{u} u$ and $\gamma_{\|}=\sqrt{1+p_{\|}^{2}}$. This distribution is log-concave with mode

$$
p_{m}=\frac{p_{u}}{A}\left(1+\sqrt{u^{2}+A^{2}}\right)
$$

and the algorithm of the Appendix will generate variates of $p_{\|}$.

Given a $p_{\|}$variate, a $p_{\perp}=\left|\mathbf{p}_{\perp}\right|$ variate conditional on it can be found. Writing $p_{s}=p_{\perp} / \sqrt{1+p_{\|}^{2}}$ the conditional distribution is

$$
f\left(p_{s} \mid p_{\|}\right)=p_{s} e^{-A\left(\gamma_{u} \sqrt{1+p_{\|}^{2}} \sqrt{1+p_{s}^{2}}-\gamma_{u} u p_{\|}-1\right)}
$$

which is equivalent to

$$
f\left(p_{s} \mid p_{\|}\right)=p_{s} e^{-A\left[\left(p_{\|}-p_{u}\right)^{2}+\gamma_{\|}^{2} \gamma_{u}^{2} p_{s}^{2}\right] /\left(\gamma_{\|} \gamma_{u} \gamma_{s}+p_{\|} p_{u}+1\right)}
$$

The mode of $f$, which is again log-concave, is

$$
p_{m}^{2}=\frac{1}{2\left(A \gamma_{u} \gamma_{\|}\right)^{2}}\left(1+\sqrt{1+\left(2 A \gamma_{u} \gamma_{\|}\right)^{2}}\right)
$$

With this background, the procedure for generating the variates of a drifting relativistic Maxwellian is: (1) Generate a variate for $p_{\|}$using the distribution of equation [11. (2) For each $p_{\|}$variate, attempt to generate a $p_{s}$ variate from the distribution of equation 14 (if the attempt fails, discard $p_{\|}$and return to step 1); (3) With $p_{\perp}=p_{s} \sqrt{1+p_{\|}^{2}}$ and a variate $\Theta$ uniformly distributed on $[0,2 \pi]$, generate the individual perpendicular variates via $p_{\perp} \cos \Theta$ and $p_{\perp} \sin \Theta$; (4) If necessary, transform from parallel/perpendicular coordinates to the desired coordinate system.

In order to check this procedure, it is useful to establish a one-dimensional version of the distribution of equation 9. To do so, express $\mathbf{p}$ in spherical coordinates, integrate over solid angle, and drop unnecessary scaling factors:

$$
f(p)=p^{2} e^{-A\left(\gamma_{u} \sqrt{1+p^{2}}-1\right)} \int_{-1}^{1} e^{A p_{u} p \cos \theta} \mathrm{d}(\cos \theta)
$$
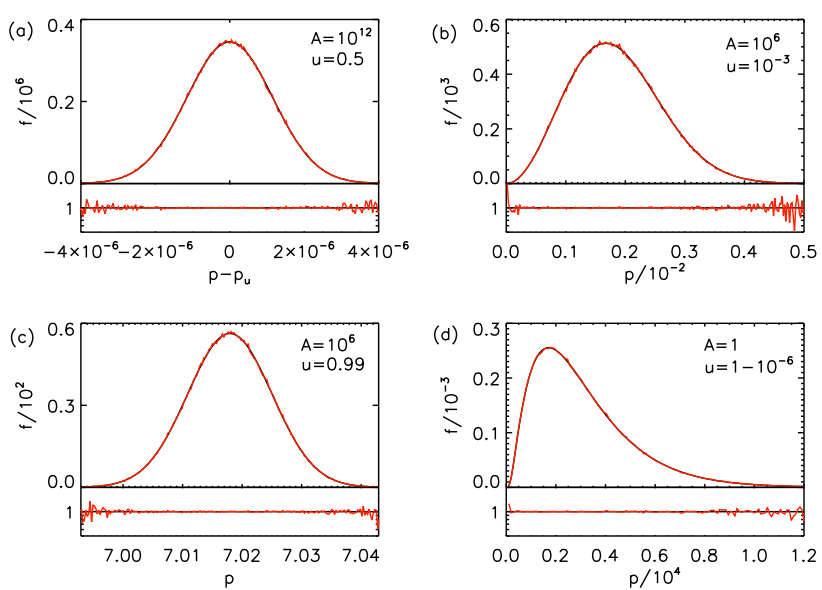

FIG. 2. Comparison, in the same format as Figure 1 between the theoretical distribution of $p$ from equation 18 and the generated random variates for drifting relativistic Maxwellians with different values of $A$ and $u$. In panel (a), $p_{u}=\gamma_{u} u \approx 0.577350$. Note the different axis scales for each panel.

The result,

$$
f(p)=p \frac{\sinh \left(A p_{u} p\right)}{A p_{u}} e^{-A\left(\gamma_{u} \sqrt{1+p^{2}}-1\right)}
$$

reduces, as it must, to equation 5 when $u=0$.

Figure 2 compares the generation of variates from distributions with four combinations of $A$ and $u$ with the theoretical form given by equation 18, For each combination, $10^{6}$ attempts were made to generate $p_{\|}$and, if successful, $p_{\perp}$. The resulting total efficiency of $77-80 \%$ depends only weakly on the parameters with small $A$ (as in the stationary case) and small $u$ yielding slightly higher values, reflecting the slightly better fits of the rejection method's bounding functions in those cases. In panels (a) and (c) the momentum corresponding to the drift, $p_{u}=\gamma_{u} u$, is larger than the typical thermal momentum and, as a consequence, the particle momenta are distributed nearly symmetrically about $p_{u}$. Panels (b) and (d), in contrast, display cases where the drift has minimal effect and the curves more resemble those of Figure 1 .

The procedure outlined in this section still works when $u=0$, albeit more slowly and less efficiently than that of Section II because it requires a second, nested call of the variate-generating algorithm. Interestingly, there exists an analytic expression that, given a single uniformly distributed variate, always returns a variate of $p_{s}=p_{\perp} / \sqrt{1+p_{\|}^{2}}$ from the $f$ of equation 14. Such a formula can be found whenever the inverse function of the integral of $f$ is known; in this case it can be written in terms of the Lambert $W$ function, for which the defining equation is $x=W(x) \exp [W(x)]$ (i.e., $W(x)$ is the inverse 
function of $\left.y=x e^{x}\right)$. The solution is

$$
\gamma_{s}=-\frac{1}{A \gamma_{\|} \gamma_{u}}\left(1+W_{-1}\left[\left(-1-A \gamma_{\|} \gamma_{u}\right) e^{\left(-1-A \gamma_{\|} \gamma_{u}\right)} U\right]\right)
$$

where $U$ is a uniformly distributed variate on $[0,1]$. $W(x)$ is multi-valued and $W_{-1}$ refers to the lower branch which, for real $x$, is only defined on the interval $[-1 / e, 0)$ with $W_{-1}(-1 / e)=-1$ and $W_{-1}\left(x \rightarrow 0^{-}\right) \rightarrow-\infty$. Equation 19 can be further simplified by noting that $W_{-1}(x)=$ $\log (-x)-\log \left(-W_{-1}(x)\right)$, but even in that form remains ill-suited for numerical work in the large $A$ limit.

\section{ACKNOWLEDGMENTS}

We thank J. Dahlin for helpful discussions and NSF grant PHY1102479.

\section{Appendix}

The algorithm below, originally described in Devroye 13 and reproduced here in a slightly altered and simpler form, generates random variates for a log-concave distribution function $f$ (one satisfying $(\log f)^{\prime \prime} \leq 0$ ) by applying the rejection method ${ }^{15}$ to a piecewise-continuous upper bound constructed of three functions: a constant intersecting the mode of $f$ and two exponential tails. Preliminary calculations are necessary to find the points $p_{-}$and $p_{+}$at which the three bounding curves meet; it can be shown that the optimal choices satisfy $f\left(p_{ \pm}\right)=f\left(p_{m}\right) / e$, where $p_{m}$ is the distribution's mode. Every $f$ considered in this work is a wellbehaved uni-modal function and standard root-finding methods (applied, for computational ease, to the equations $\left.\log \left[f\left(p_{ \pm}\right) / f\left(p_{m}\right)\right]+1=0\right)$ quickly converge to $p_{ \pm}$. Note that because $f$ only appears in ratios, even during the preliminary root-finding, its normalization is irrelevant.

Although the algorithm is not guaranteed to generate a variate on each iteration, its efficiency for an arbitrary $f$ can be shown to lie between $\approx 30$ and $100 \%$. Generation of $10^{6}$ variates takes $\mathcal{O}(0.1)$ seconds in unoptimized Fortran. For additional algorithmic details, derivations, a discussion of efficiency, and alternative methods, see Devroye ${ }^{13}$.

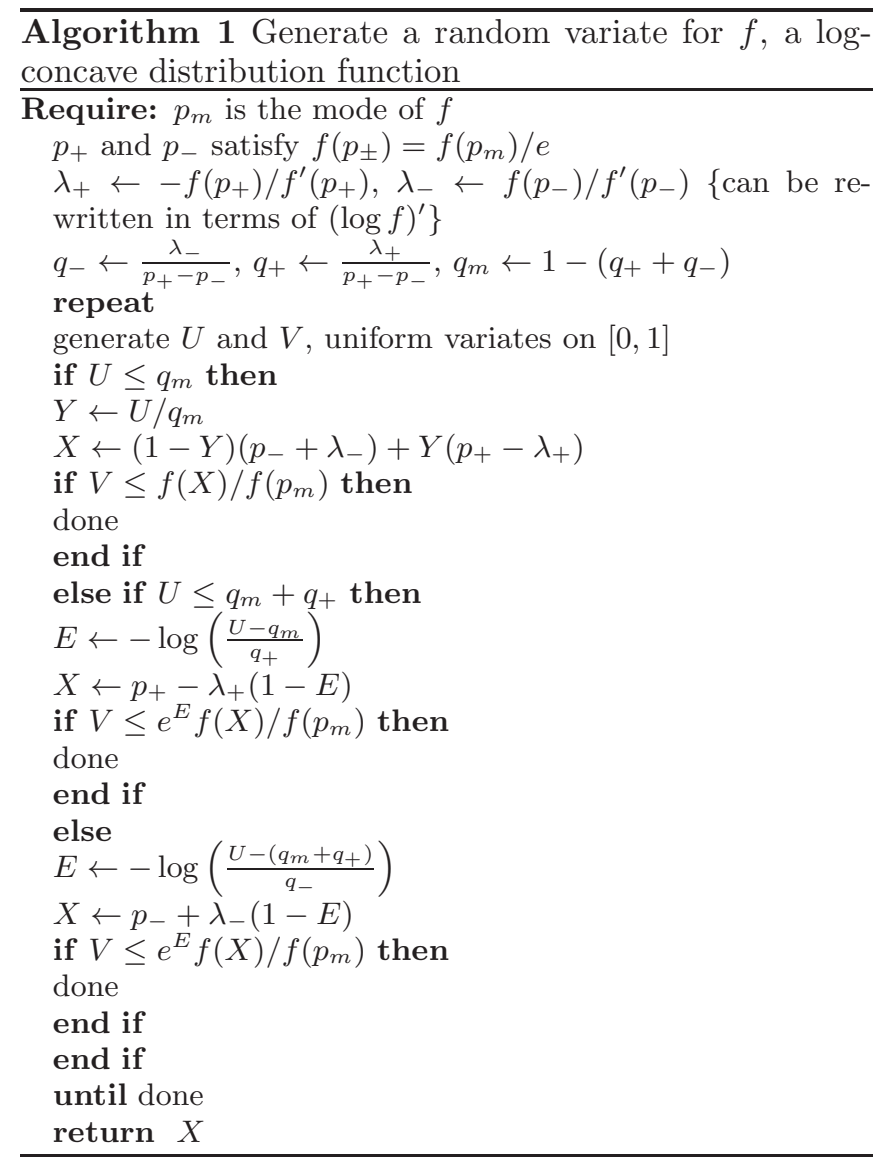

${ }^{1}$ G. Marsaglia and T. A. Bray, SIAM Review 6, 260 (1964).

${ }^{2}$ F. Jüttner, Ann. d. Phys. 339, 856 (1911).

${ }^{3}$ E. Lehmann, J. Math. Phys. 47 (2006), 10.1063/1.2165771

${ }^{4}$ G. Chacón-Acosta, L. Dagdug, and H. A. Morales-Técotl, Phys. Rev. E 81, 021126 (2010), 10.1103/PhysRevE.81.021126

${ }^{5}$ R. A. Treumann, R. Nakamura, and W. Baumjohann, Ann. Geophys. 29, 1259 (2011)

${ }^{6}$ J. L. Synge, The Relativistic Gas (North-Holland Pub. Co., 1957).

${ }^{7}$ O. Barndorff-Nielsen, Proc. R. Soc. Lond. A 353, 401 (1977).

${ }^{8}$ O. Barndorff-Nielsen, Scand. J. Statist. 9, 43 (1982).

${ }^{9} \mathrm{P}$. Blaesild and J. Jensen, "Multivariate distributions of hyperbolic type," in Statistical distributions in scientific work (Trieste, 1980), NATO ASI Series C Mathematical and Physical Sciences - Advanced Study Institute, Vol. 4 (D. Reidel Publishing Group, 1981) pp. $45-66$.

${ }^{10}$ A. C. Atkinson, SIAM J. Sci. Stat. Comput. 3, 502 (1982).

${ }^{11}$ L. A. Pozdnyakov, I. M. Sobol, and R. A. Syunyaev, Sov. Astron. 21, 708 (1977).

${ }^{12}$ L. A. Pozdnyakov, I. M. Sobol, and R. A. Syunyaev, Soviet Scientific Reviews, Section E: Astrophysics and Space Physics Reviews 2, 189 (1983).

${ }^{13}$ L. Devroye, "Non-uniform random variate generation," (Springer-Verlag, 1986) Chap. 7, pp. 286-357, available at http://luc.devroye.org/rnbookindex.html.

${ }^{14}$ R. E. Knop, Communications of the ACM 13, 326 (1970).

${ }^{15}$ W. H. Press, S. A. Teukolsky, W. T. Vetterling, and B. P. Flannery, "Numerical recipes in Fortran 77," (Cambridge University Press, 1992) Chap. 7.3, pp. 281-286. 